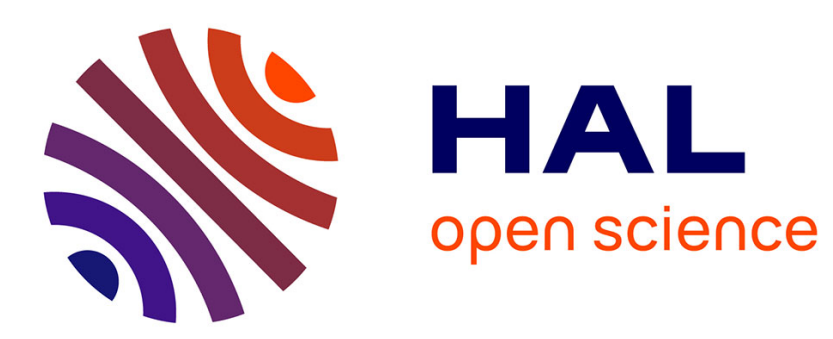

\title{
Effets photovoltaïques de transitions interbandes dans des structures métal/photoconducteur/métal
}

\author{
A. Piel, H. Murray
}

\section{To cite this version:}

A. Piel, H. Murray. Effets photovoltaïques de transitions interbandes dans des structures métal/photoconducteur/métal. Revue de Physique Appliquée, 1976, 11 (3), pp.397-402. 10.1051/rphysap:01976001103039700 . jpa-00244074

\section{HAL Id: jpa-00244074 https://hal.science/jpa-00244074}

Submitted on 1 Jan 1976

HAL is a multi-disciplinary open access archive for the deposit and dissemination of scientific research documents, whether they are published or not. The documents may come from teaching and research institutions in France or abroad, or from public or private research centers.
L'archive ouverte pluridisciplinaire HAL, est destinée au dépôt et à la diffusion de documents scientifiques de niveau recherche, publiés ou non, émanant des établissements d'enseignement et de recherche français ou étrangers, des laboratoires publics ou privés. 
Classification

Physics Abstracts

8.860

\title{
EFFETS PHOTOVOLTAÏQUES DE TRANSITIONS INTERBANDES DANS DES STRUCTURES MÉTAL/PHOTOCONDUCTEUR/MÉTAL
}

\author{
A. PIEL et H. MURRAY \\ Laboratoire d'Electronique et d'Automatique \\ U. E. R. des Sciences et Techniques \\ B. P. no 4006, 76077 Le Havre Cedex, France \\ (Reçu le 16 juin 1975, révisé le 2 décembre 1975, accepté le 7 janvier 1976)
}

\begin{abstract}
Résumé. - Les effets photovoltaïques apparaissant dans les structures $\mathrm{Al} / \mathrm{ZnS} / \mathrm{Au}$ ont montré l'existence de deux phénomènes [1] dont l'origine a été attribuée à des interactions électrons photons soit par transitions interbandes, soit par photoexcitation Poole-Frenkel.

Les valeurs du rendement énergétique obtenues dans le domaine des longueurs d'onde provoquant les transitions interbandes, nous ont fait apparaître l'intérêt d'une étude systématique des structures Métal $_{1} /$ photoconducteur/Métal 2 lorsque l'épaisseur du photoconducteur est comprise entre 100 et $1000 \AA$.

Nous nous proposons, dans cette étude, de rechercher une formulation théorique de ces effets à partir des équations de continuité [2] qui sont à la base des études de photoconduction. La formulation théorique ainsi obtenue est cohérente avec les effets expérimentaux observés dans les structures $\mathrm{Al} / \mathrm{ZnS} / \mathrm{Au}$ et $\mathrm{Al} / \mathrm{CdS} / \mathrm{Au}$.
\end{abstract}

\begin{abstract}
Photovoltaic effects, which appear in $\mathrm{Al} / \mathrm{ZnS} / \mathrm{Au}$ structures have shown the existence of two phenomena [1] which can be attributed either to interband transitions or to Poole-Frenkel photoexcitation.

In the range of wave-length which can induce interband transitions, we obtain a value for the efficiency of energy transfer that is sufficiently high to warrant a systematic study of $\mathrm{Metal}_{1} / \mathrm{photo}$ conductor/Metal 2 structures when the photoconductor thickness varies from 100 to $1000 \AA$.

We propose, in this study, a theoretical formulation of these effects obtained from basic continuity equations [2]. This theoretical formulation is in good agreement with the experimental results observed in $\mathrm{Al} / \mathrm{ZnS} / \mathrm{Au}$ and $\mathrm{Al} / \mathrm{CdS} / \mathrm{Au}$ structures.
\end{abstract}

1. Introduction. - On appelle effet photovoltaïque l'apparition d'une tension ou d'un courant dans un matériau soumis à une radiation lumineuse et réalisant la conversion d'énergie lumineuse en énergie électrique. Pour qu'un tel effet se produise, il est nécessaire que d'une part des porteurs puissent transiter dans la bande interdite, et d'autre part, qu'un champ électrique interne éventuellement affecté de variations dimensionnelles [4] entraîne le mouvement de ces porteurs en l'absence de tension appliquée. Ce phénomène se trouve facilement réalisé dans les jonctions $\mathrm{P}-\mathrm{N}$ dont les applications pratiques sont bien connues [5]. Mais ils apparaissent aussi dans certaines structures tunnel [6] ou dans des structures sandwich à partir de l'émission d'électrons aux interfaces Métal-Isolant ou MétalSemiconducteur.

La réalisation de structures $\mathrm{Al} / \mathrm{ZnS} / \mathrm{Au}$ dont l'épaisseur du diélectrique se situe entre 100 et $1000 \AA$ permet d'obtenir, à l'intérieur du diélectrique, dont l'homogénéité macroscopique a été montrée par ailleurs [7], un champ électrique élevé provoquant le transport des porteurs photoexcités, tel que le mouvement d'électrons en court-circuit soit dirigé de l'électrode d'or vers l'électrode d'aluminium, lorsque l'épaisseur est supérieure à $240 \AA ̊$.

De telles structures présentent un intérêt particulier dû à leur fiabilité [7] ; dès lors que les conditions technologiques nécessaires à l'élaboration de couches très minces de diélectrique ont pu être maîtrisées.

Les propriétés électroniques et photoélectroniques de ces structures ont déjà été décrites et des interprétations ont été données, cohérentes avec les résultats expérimentaux $[8,9]$. Nous nous proposons, dans cette étude, de donner une formulation théorique des effets de photoconduction de transitions interbandes, à partir des équations fondamentales de continuité des densités de courant; les hypothèses simplificatrices étant justifiées par les résultats expérimentaux obtenus.

2. Formulation théorique de la relation de photoconduction. - 2.1 EQUATIONS FONDAMENTALES ET HYPOTHÈSES SIMPLIFICATRICES. - Le modèle de bande 
théorique d'une structure $\mathrm{Mé} \mathrm{Il}_{1} / \mathrm{photoconducteur/}$ Métal $_{2}$ est représenté sur la figure 1 . L'origine des abscisses est prise à l'interface photoconducteur/ Métal $_{2}$ (semi-transparent).

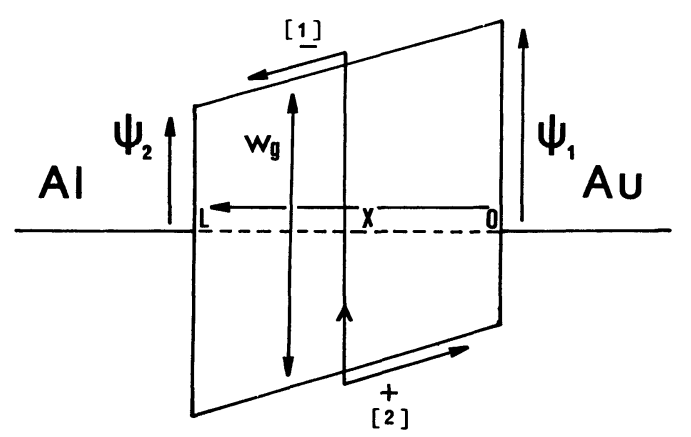

FIG. 1. - Diagramme d'énergie théorique d'une structure Métal/photoconducteur/Métal. $\Psi_{1,2}$ travaux de sortie Métal/photoconducteur. $W_{\mathbf{g}}$ Largeur de la bande interdite. $X$ Abscisse. [1] Mouvement des porteurs négatifs. [2] Mouvement des porteurs positifs.

Nous poserons par la suite :

$n(x)$ : concentration d'électrons

$p(x)$ : concentration de trous

$J_{\mathrm{n}}(x)$ : densité de courant d'électrons

$J_{\mathrm{p}}(x)$ : densité de courant de trous

$g(x)$ : nombre de paires électrons-trous créées par le flux lumineux

$R_{\mathrm{n}}(x)$ : taux de recombinaison des électrons

$R_{\mathrm{p}}(x)$ : taux de recombinaison des trous.

L'équilibre entre les générations et les recombinaisons de porteurs s'exprime à partir des relations : (2)

$$
R_{\mathrm{n}}(x)=\frac{n(x)}{\tau_{\mathrm{n}}} \quad \text { et } \quad R_{\mathrm{p}}(x)=\frac{p(x)}{\tau_{\mathrm{p}}}
$$

Lorsque le régime d'équilibre s'est établi, soit

$$
\frac{\mathrm{d} n(x)}{\mathrm{d} t}=\frac{\mathrm{d} p(x)}{\mathrm{d} t}=0
$$

la résolution des équations de continuité à partir des relations entre les densités de courant et les densités de porteurs libres permet d'exprimer les densités de courant.

Nous utiliserons, pour cela, les hypothèses simplificatrices suivantes, justifiées expérimentalement :

a) Compte tenu de la faible épaisseur du diélectrique vis-à-vis de la longueur d'absorption du rayonnement lumineux, on peut admettre l'indépendance de $g(x)$ en fonction de $x$. Dans la suite des calculs, nous poserons donc $g(x)=g=k F$ ( $F$ : lumineux).

b) Nous n'introduisons pas de niveaux intermédiaires dans la bande interdite car ceux-ci n'apparaissent ni dans les phénomènes de conduction [8], ni dans les phénomènes de photoconduction [9].
Nous avons montré en effet, lorsque l'énergie des photons est inférieure à la largeur de bande, l'existence d'une photoexcitation Poole-Frenkel identique aux effets observés par Jonscher et Ansari dans le monoxyde de silicium [10]. Comme le font remarquer ces auteurs, une photoexcitation d'électrons au-dessus de la barrière entourant les centres coulombiens, provoque une variation continue des caractéristiques de photoconduction en fonction de l'énergie $\mathrm{h} v$ des photons incidents sans apparition de seuil énergétique, alors que des transitions à l'intérieur de la bande interdite à partir de niveaux pièges provoquerait une variation non continue avec seuil ou palier.

\subsection{Equations Relatives aUX DENSITÉS DE COURANT.} - Par substitution des équations de continuité [2] dans les équations relatives aux densités de courant, nous obtenons les équations différentielles suivantes :

$$
\begin{aligned}
& D_{\mathrm{n}} \tau_{\mathrm{n}} \frac{\mathrm{d}^{2} J_{\mathrm{n}}}{\mathrm{d} x^{2}}+\mu_{\mathrm{n}} \tau_{\mathrm{n}} E \frac{\mathrm{d} J_{\mathrm{n}}}{\mathrm{d} x}-J_{\mathrm{n}}(x)=-e \mu_{\mathrm{n}} \tau_{\mathrm{n}} g E \\
& D_{\mathrm{p}} \tau_{\mathrm{p}} \frac{\mathrm{d}^{2} J_{\mathrm{p}}}{\mathrm{d} x^{2}}-\mu_{\mathrm{p}} \tau_{\mathrm{p}} E \frac{\mathrm{d} J_{\mathrm{p}}}{\mathrm{d} x}-J_{\mathrm{p}}(x)=-e \mu_{\mathrm{p}} \tau_{\mathrm{p}} g E .
\end{aligned}
$$

Ces deux équations sont du second ordre, linéaires, à cœefficients constants, avec un second membre indépendant de $x$.

Les solutions de ces équations se mettent sous la forme simplifiée :

et

$$
J_{\mathrm{n}}(x)=J_{\mathrm{n} 0}(x)+e \mu_{\mathrm{n}} \tau_{\mathrm{n}} g E
$$

$$
J_{\mathrm{p}}(x)=J_{\mathrm{p} 0}(x)+e \mu_{\mathrm{p}} \tau_{\mathrm{p}} g E .
$$

$J_{\mathrm{n} 0}(x)$ et $J_{\mathrm{p} 0}(x)$ représentant respectivement les densités d'électrons et de trous à flux lumineux nul s'obtiennent lorsque les seconds membres des éq. (5) et (6) sont nuls $(g=0)$. Nous pouvons remarquer que ces équations sont alors identiques aux équations donnant la densité de courant d'obscurité qui s'écrit, lorsque le champ appliqué est faible [8] :

$$
\begin{gathered}
J_{\mathrm{ob}}=\sigma_{\mathrm{ob}} E=J_{\mathrm{n} 0}(x)+J_{\mathrm{p} 0}(x) \\
\operatorname{avec}\left\{\begin{array}{l}
J_{\mathrm{n} 0}(x)=A \exp r_{1} x+B \exp r_{2} x \\
J_{\mathrm{p} 0}(x)=C \exp r_{1}^{\prime} x+D \exp r_{2}^{\prime} x \\
\text { Solutions des équations sans second membre } \\
\text { associées. }
\end{array}\right.
\end{gathered}
$$

Néanmoins, lorsqu'on applique un flux lumineux $F$, les conditions aux limites en $x=0$ et $x=L$ ne sont pas identiques aux conditions aux limites d'obscurité et modifient les constantes d'intégration $(A, B, C, D)$ intervenant dans le calcul du courant à flux lumineux nul. Ceci introduit une valeur de $\sigma_{0}$ différente de $\sigma_{0 \mathrm{~b}}$ comme on l'observe expérimentalement (Fig. 2).

Par contre, la linéarité des caractéristiques couranttension (Fig. 2) dans le domaine de tension et de longueur d'onde étudié expérimentalement nous permet 


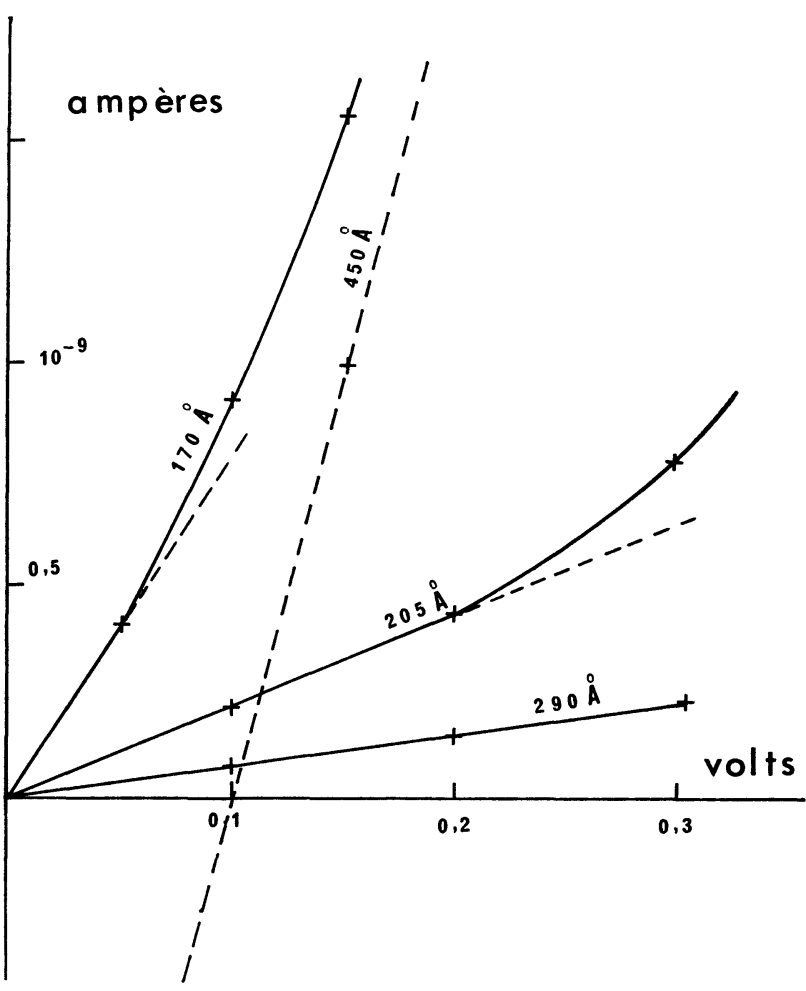

Fig. 2. - Caractéristiques linéaires courant-tension. ( - ) courant d'obscurité linéaire pour un champ électrique inférieur à $10^{5} \mathrm{~V} / \mathrm{cm}$. (- -) courant photoélectrique linéaire de transitions interbandes.

de considérer une conductivité indépendante du champ électrique appliqué tant pour $\sigma_{0}, \sigma_{0 \mathrm{~b}}$ que $\sigma_{\mathrm{ph}}$.

L'introduction d'une conductivité $\sigma_{0}$ permet d'écrire la densité de courant totale sous la forme

$$
J_{\mathrm{t}}=\sigma_{0} E+e g\left(\mu_{\mathrm{n}} \tau_{\mathrm{n}}+\mu_{\mathrm{p}} \tau_{\mathrm{p}}\right) E .
$$

Le facteur eg $\left(\mu_{\mathrm{n}} \tau_{\mathrm{n}}+\mu_{\mathrm{p}} \tau_{\mathrm{p}}\right)$ représente la photoconductivité normale telle qu'elle est donnée par Bube [11], ce qui permet d'écrire :

avec

$$
J_{\mathrm{t}}=\left(\sigma_{0}+\sigma_{\mathrm{ph}}\right) E
$$

$$
\sigma_{\mathrm{ph}}=e g\left(\mu_{\mathrm{n}} \tau_{\mathrm{n}}+\mu_{\mathrm{p}} \tau_{\mathrm{p}}\right) \text {. }
$$

3. Structures - métal 1 / Photoconducteur/métal 2 . Lorsque les deux métaux entourant le photoconducteur sont différents, il existe à l'intérieur de celui-ci un champ électrique interne théorique : $E=\Psi_{1}-\Psi_{2} / e d$. En fait, le champ électrique expérimental peut être différent en raison de la distorsion de la bande de conduction par des phénomènes de volume dont une explication est donnée par ailleurs [12].

Par la suite, nous désignerons par $E_{\mathrm{i}}$ le champ électrique réel en l'absence de champ appliqué.

Si la distorsion du champ électrique interne résulte de phénomènes de volume induits par la photoionisation des centres coulombiens [13], nous n'avons par contre jamais observé de variations de capacité supérieures à la précision des mesures [14], même lorsque ces struc- tures sont fortement éclairées par un flux lumineux ultra-violet.

Ce phénomène entraîne l'absence de modifications des phénomènes électrostatiques des structures, donc un champ électrique interne, indépendant de $x$, mais modulé par des phénomènes dimensionnels [12].

Si on appelle $E_{0}$ le champ appliqué, la densité de courant totale se met sous la forme :

$$
J_{\mathrm{T}}=\left(\sigma_{0}+\sigma_{\mathrm{ph}}\right) E_{\mathrm{i}}+\left(\sigma_{0}+\sigma_{\mathrm{ph}}\right) E_{0} .
$$

La densité de courant de court-circuit s'obtient en posant $E_{0}=0$, soit :

$$
J_{\text {pho }}=\left(\sigma_{0}+\sigma_{\text {ph }}\right) E_{\mathrm{i}} .
$$

Le champ électrique en circuit ouvert s'obtient en posant $J_{\mathbf{T}}=0$, soit :

$$
E_{\text {pho }}=-E_{\mathrm{i}} \text {. }
$$

En remplaçant les densités de courant par les courants et les conductivités par les conductances, la relation théorique entre le courant de court-circuit et la tension photovoltaïque devient :

$$
I_{\mathrm{pho}}=-\left(G_{0}+G_{\mathrm{ph}}\right) V_{\mathrm{pho}} .
$$

La formulation mathématique ainsi obtenue montre que ces structures sont des générateurs d'un courant produit par le transport des porteurs sous l'effet d'un champ électrique interne; la tension photovoltaïque en circuit ouvert qui s'établit est celle qui provoque un courant de conduction et de photoconduction opposé au courant de court-circuit.

4. Applications aux structures $\mathrm{Al} / \mathrm{ZnS} / \mathrm{Au}$ et Al/CdS/Au. - 4.1 MéthODes EXPÉRIMENTALes. Les méthodes expérimentales d'élaboration des structures sandwich ont été précédemment décrites $[7,8]$. La méthode de dépôt utilisée (pulvérisation cathodique H. F.), permet, en raison des qualités mécaniques des couches déposées, de résoudre les obstacles technologiques dont dépend la bonne reproductibilité des résultats pour de faibles épaisseurs de diélectrique.

Par contre, par cette méthode, le piégeage de particules provenant du gaz de décharge, inhérent à ce procédé de dépôt, induit des propriétés spécifiques de conduction et de photoconduction introduisant un effet photovoltaïque Poole-Frenkel de type nouveau [8]. Cet effet, de rendement énergétique faible est entièrement masqué par l'effet photovoltaïque de transitions interbandes dès que l'énergie lumineuse est supérieure à la largeur de bande interdite [1].

Les expérimentations ont lieu in situ dans l'enceinte à vide, et après formation et stabilisation, la structure est éclairée à travers un hublot, par un flux lumineux monochromatique issu d'un monochromateur à prisme (quartz ou flint selon le domaine de longueur d'onde étudié) associé à une lampe quartz-iode de $150 \mathrm{~W}$.

4.2 Résultats EXPéRIMENTAUX. - Dans le domaine des transitions interbandes, les résultats 
expérimentaux, représentés sur la figure $3 a$ montrent les caractéristiques suivantes :

a) le courant de court-circuit est proportionnel au flux lumineux,

b) la tension photovoltaïque présente une croissance non linéaire lente à partir de $10 \mu \mathrm{W} / \mathrm{cm}^{2}$,

c) le rapport : $-I_{\mathrm{pho}} / V_{\mathrm{pho}}$ est fonction linéaire du flux lumineux en bonne cohérence avec l'équation théorique :

$$
I_{\mathrm{pho}}=-\left(G_{0}+G_{\mathrm{ph}}\right) V_{\mathrm{pho}} .
$$
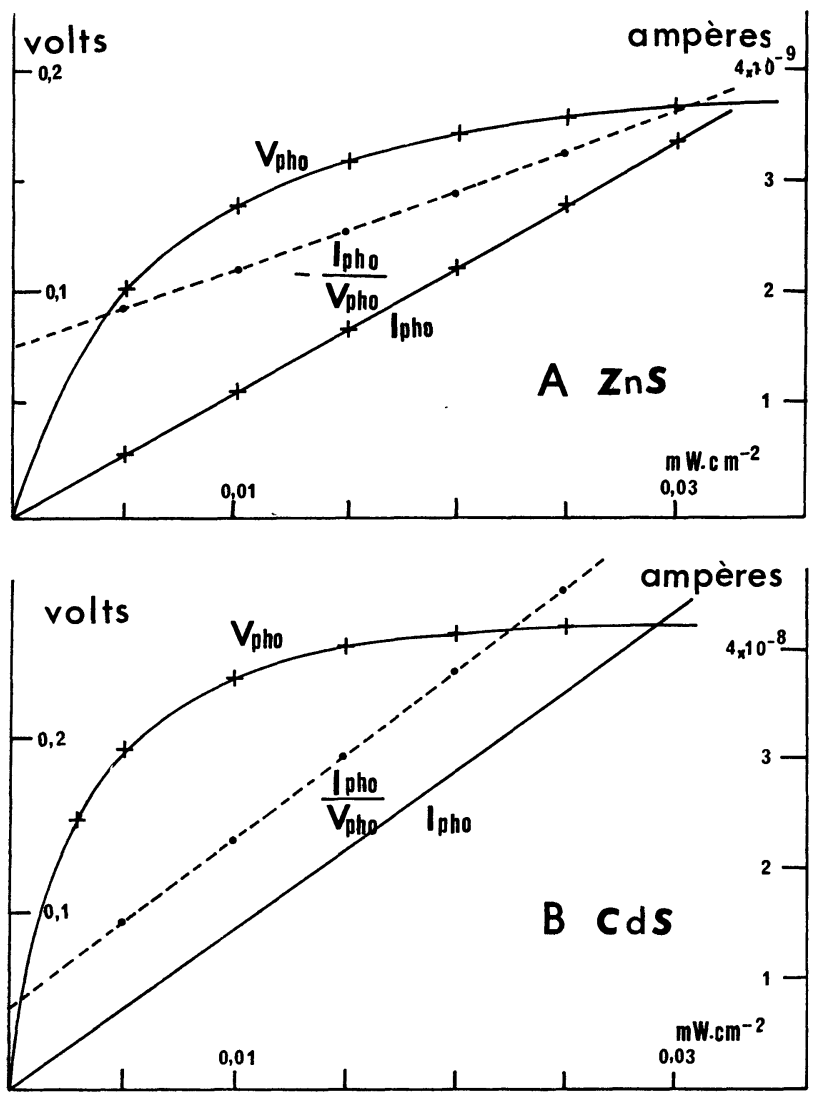

FIg. 3. - Représentation du courant de court-circuit ( $\left.I_{\mathrm{pho}}\right)$ de la tension photovoltaique $\left(V_{\text {pho }}\right)$ et de la conductance $G_{0}+G_{\mathrm{ph}}$ en fonction du flux lumineux. a) structure $\mathrm{Al} / \mathrm{ZnS} / \mathrm{Au}$ b) structure $\mathrm{Al} / \mathrm{CdS} / \mathrm{Au}$.

L'extrapolation de la droite $\left(G_{0}+G_{\mathrm{ph}}\right)$ à flux lumineux nul, donne la valeur de $G_{0}(\lambda)$ qui se trouve être effectivement supérieure à la conduction d'obscurité et dépendante des conditions aux limites en $x=0$ et $x=L$.

Des expériences récentes, effectuées sur des structures $\mathrm{Al} / \mathrm{CdS} / \mathrm{Au}$ ont montré des résultats expérimentaux qualitativement identiques, mais dans ce cas, le. niveau d'énergie lumineuse nécessaire pour provoquer des transitions interbandes se trouve situé dans le domaine de rayonnement visible (supérieure à $2,1 \mathrm{eV}$ ) (Fig. 3b).

Ces résultats confirment les hypothèses avancées lors de l'étude des structures $\mathrm{Al} / \mathrm{ZnS} / \mathrm{Au}$. Nous remar- quons en effet que la tension photovoltaïque obtenue pour un niveau donné d'illumination est d'un même ordre de grandeur pour les deux types de structures, mais que le rapport courant de court-circuit/flux lumineux est plus important dans le cas du sulfure de cadmium comme le laisse prévoir les propriétés photoconductrices des deux sulfures.

5. Etude du rendement énergétique. - 5.1 FoRMULATION DU RENDEMENT ÉNERGÉTIQUE. - Les structures étudiées sont susceptibles de fournir une puissance électrique lorsqu'elles débitent sur une résistance externe passive. Nous définirons cette puissance électrique à partir du produit :

$$
P_{\mathrm{e}}=I_{\text {pho }} \times V_{\text {pho }} .
$$

La puissance maximum fournie se situant à $P_{\mathrm{e}} / 4$ en raison du caractère quasi linéaire des variations $(I, V)$.

D'après les expressions de $I_{\text {pho }}$ et $V_{\text {pho }}$, on obtient alors :

$$
P_{\mathrm{e}}=\left(G_{0}+G_{\mathrm{ph}}\right) V_{\mathrm{pho}}^{2} .
$$

Par unité de volume du photoconducteur situé entre les électrodes, cette puissance s'exprime par :

$$
P_{\mathrm{e}(v)}=\left(\sigma_{0}+\sigma_{\mathrm{ph}}\right) E_{\mathrm{i}}^{2} .
$$

Le rendement énergétique est défini par le rapport : $\rho=P_{\mathrm{e}} / F, F$ étant le flux lumineux exprimé en watts.

L'expression de $P_{\mathrm{e}}$ permet d'écrire $\rho$ sous la forme :

$$
\rho=\frac{\left(\sigma_{0}+\sigma_{\mathrm{ph}}\right) E_{\mathrm{i}}^{2}}{F} .
$$

Cette expression montre que le rendement énergétique reste constant pour les valeurs du flux lumineux supérieures à $0,03 \mathrm{~mW} / \mathrm{cm}^{2}$ correspondant à $\sigma_{\mathrm{ph}} \gg \sigma_{0}$. Cette valeur peu élevée du flux lumineux présente un intérêt dans les applications pratiques puisqu'elle permet d'obtenir des convertisseurs linéaires : flux lumineux/courant photoélectrique, particulièrement dans le cas du sulfure de cadmium.

Le rendement énergétique est essentiellement lié à la photoconductivité du matériau dès que la valeur de seuil du flux lumineux est dépassée, les valeurs expérimentales de $E_{\mathbf{i}}$ étant pratiquement identiques dans les mêmes domaines d'épaisseur.

L'étude de l'expression du rendement énergétique présente alors un intérêt si on connaît la photoconductivité. Celle-ci dépend pour le matériau étudié, des caractéristiques de conduction (mobilité, durée de vie), ainsi que de la largeur de bande interdite.

5.2 ETUDE EXPÉRIMENTALE ET THÉORIQUE DU RENDEMENT ÉNERGÉTIQUE. - La figure 4 montre les variations du rendement énergétique en fonction de la longueur d'onde du rayonnement incident sur des structures $\mathrm{Al} / \mathrm{CdS} / \mathrm{Au}$. La brusque décroissance de $\rho$ 


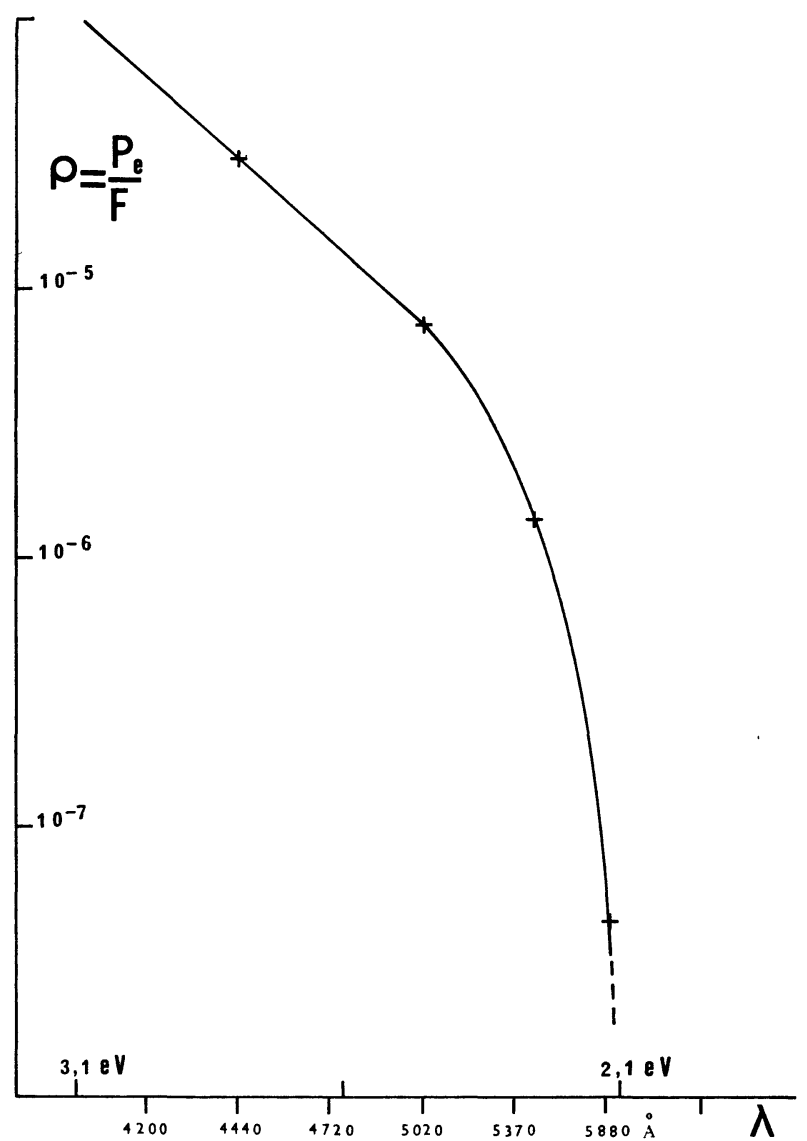

Fig. 4. - Représentation du rendement énergétique $\rho=P_{\mathrm{e}} / F$ en fonction de la longueur d'onde $\lambda$ du rayonnement incident.

au voisinage du niveau de bande interdite, confirme l'hypothèse d'un effet photovoltaiqque induit par transitions interbandes.

Dans cette hypothèse, les cellules de CdS se révèlent mieux adaptées dans le cadre d'une utilisation pratique dans le domaine du spectre visible et celle-ci laisse prévoir un meilleur rendement énergétique avec un photoconducteur de plus faible largeur de bande interdite.

A partir des résultats expérimentaux exprimés par les figures $3 a$ et $3 b$, il est possible de comparer les valeurs du rendement obtenues, avec les valeurs théoriques que laissent prévoir la formulation mathématique, en relation avec le modèle de bande théorique.

La puissance électrique s'exprime en effet, dès que $\sigma_{\mathrm{ph}} \gg \sigma_{0}$ par :

$$
P_{\mathrm{e}}=\sigma_{\mathrm{ph}} E_{\mathrm{i}}^{2}
$$

Le champ électrique interne, théoriquement identique pour les deux types de structure, permet d'écrire le rapport des puissances électriques obtenues par la formule simplifiée :

$$
\frac{\left(P_{\mathrm{e}}\right) \mathrm{CdS}}{\left(P_{\mathrm{e}}\right) \mathrm{ZnS}} \approx \frac{\left(\sigma_{\mathrm{ph}}\right) \mathrm{CdS}}{\left(\sigma_{\mathrm{h}}\right) \mathrm{ZnS}} .
$$

Pour des valeurs identiques du flux lumineux, le rapport expérimental

$$
\frac{\left(P_{\mathrm{e}}\right) \mathrm{CdS}}{\left(P_{\mathrm{e}}\right) \mathrm{ZnS}}
$$

est de l'ordre de 10.

Cette valeur, obtenue dans des conditions expérimentales identiques (mêmes paramètres d'élaboration des couches), est en fait difficile à comparer avec des estimations théoriques [15].

La photoconductivité s'écrit :

$$
\sigma_{\mathrm{ph}}=e g\left(\mu_{\mathrm{n}} \tau_{\mathrm{n}}+\mu_{\mathrm{p}} \tau_{\mathrm{p}}\right) .
$$

Les deux types de photoconducteurs envisagés (CdS et $\mathrm{ZnS}$ ) sont de type $\mathrm{n}$ lorsqu'ils ne sont pas dopés. On peut alors écrire :

$$
\sigma_{\mathrm{ph}} \simeq e g\left(\mu_{\mathrm{n}} \tau_{\mathrm{n}}\right) .
$$

Plusieurs auteurs $[15,16]$ ont montré la difficulté de considérer cette photoconductivité comme une grandeur intrinsèque du matériau puisque selon le processus envisagé, les valeurs de la mobilité et de la durée de vie sont très variables. Ceci entraîne la difficulté de définir une estimation théorique de la puissance électrique.

Nous remarquerons cependant qu'une estimation qualitative est possible si on se rapporte aux valeurs des courants d'obscurité obtenus [17]. Ces courants d'obscurité, interprétés à partir d'une émission PooleFrenkel [18], montrent une mobilité plus importante du CdS, si on se réfère aux formules de Hill [19] (Fig. 5).

$$
J=2 e N_{\mathrm{i}}(k T)^{4} \beta^{-2} \mu \exp -\frac{E_{\mathrm{i}}}{k T} \alpha^{2} \operatorname{sh} \alpha .
$$

L'aspect qualitatif du rendement énergétique obtenu

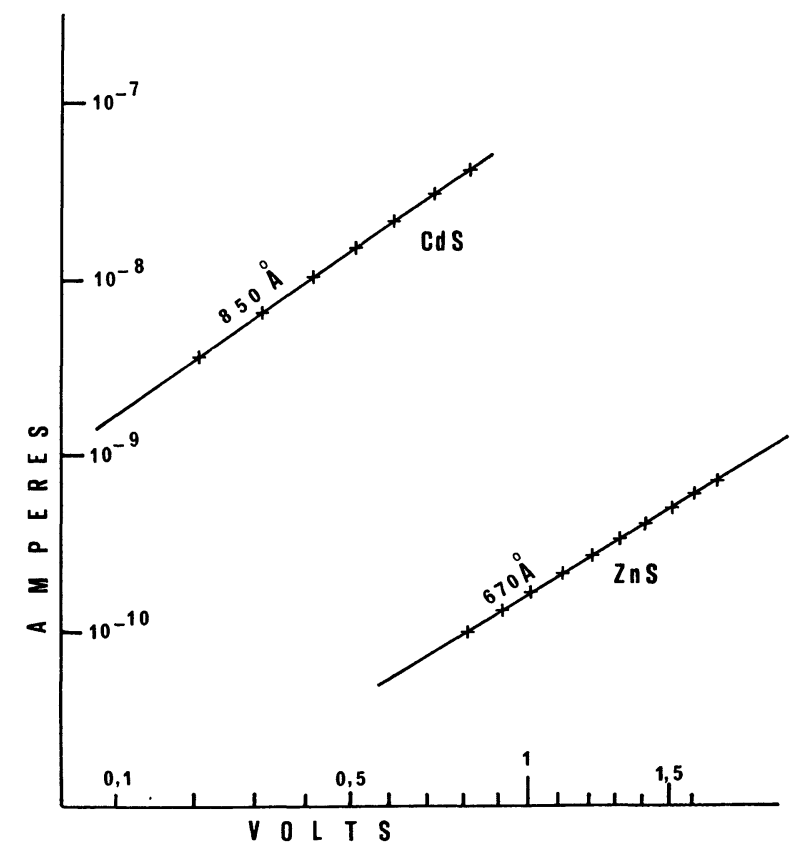

FIG. 5. - Caractéristiques d'obscurité $I=f(V)$ dans le système de coordonnées $\log I,(V)^{1 / 2}$. 
est ainsi en accord avec les prévisions théoriques qui entraînent

$$
\mu_{\mathrm{CdS}}>\mu_{\mathrm{ZnS}} \text {. }
$$

Cette propriété est particulièrement importante lors d'applications pratiques dans le domaine des convertisseurs d'énergie puisque les cellules au CdS présentent par rapport au $\mathrm{ZnS}$ une photoconductivité supérieure et un déplacement du seuil énergétique vers le maximum $\mathrm{du}$ spectre solaire.

6. Conclusion. - Les résultats expérimentaux obtenus montrent que le rendement massique est élevé, compte tenu des faibles épaisseurs de photoconducteur, ce qui permet d'envisager des applications dans deux domaines :

- les détecteurs de photons dans le domaine des longueurs d'ondes visibles ou ultra-violettes selon le photoconducteur ;
- les convertisseurs d'énergie lumineuse en énergie électrique.

Nous avons déjà souligné, dans de précédents travaux [7], l'application des propriétés spécifiques de la pulvérisation cathodique pour la mise en œuvre des capacités de forte valeur utilisables en micro-électronique. L'orientation de notre étude, vers des structures dont l'épaisseur du diélectrique est inférieure à $1000 \AA$, offre un nouveau champ d'investigation dans le domaine de l'interaction électron-photon en présence de centres coulombiens dans le diélectrique. Les résultats obtenus tant avec le sulfure de zinc que le sulfure de cadmium, montrent l'importance de cette étude systématique sur des structures Métal $_{1} /$ photoconducteur/Métal ${ }_{2}$ dont le rendement massique peut être amplifié par l'élaboration d'une répartition des structures dans un motif périodique (ce que permet la pulvérisation cathodique).

\section{Bibliographie}

[1] MURray, H. and Tosser, A., 6th International symposium on Photon Detectors (IMEKO) Siofok, Hungary, September (1974).

[2] Bube, R. H., Photoconductivity of solids (John Wiley and Sons, New York) p. 326.

[3] Moss, T. S., Burrel, G. J. and Ellis, B., Semi-conductor opto-electronics (Butherworths) 1973, 153.

[4] Ryvkin, S. M., Photoelectric effects in semi-conductors (Consultants Bureau New York) 1964, 369.

[5] RAPPAPORT, P. and Wisocki, J. J., Photoelectronic materials and devices (Van Nostrand company New York) 1965, 239-272.

[6] Tosser, A., Thèse de Doctorat d'Etat, Université de Caen (1967).

[7] Murray, H. and Tosser, A., Thin Solid Films 17 (1973) 7583.

[8] Murray, H. and Tosser, A., Thin Solid Films 22 (1974) 3744.

[9] MURray, H. and Tosser, A., Thin Solid Films 24 (1974) 165180.
[10] Jonscher, A. K. and ANSAri, A. A., Phil. mag. 23 (1971) 205-223.

[11] BUBE, R. H., Photoconductivity of solids (John Wiley and Sons, New York) 1960, p. 58.

[12] Tosser, A., Murray, H. and Arsalane, M., Thin Solid Films (à paraître, 1976).

[13] TOSSER, A. and MURRAH, Electrocomponent Science and Technology 2 (1975) 147-155.

[14] Murray, H., Thèse de Doctorat d'Etat, Université de Rouen, 1974.

[15] Cebe, J. P. et Mathieu, J. M., C. R. Hebd. Séan. Acad. Sci. Paris 270 B (1970) 1111-1114.

[16] Garron et Ihaya. C. R. Hebd. Séan. Acad. Sci. Paris 263 B (1966) 615.

[17] MuRRay, H. and Tosser, A., 3rd International Conference on thin films (I. C. T. F. 3) Budapest Hungary, 2529 August 1975.

[18] Frenkel, J., Phys. Rev. 54 (1938) 657.

[19] HiLl, R. M., Phil. Mag. 23 (1971) 59-86. 